\title{
Neuroimaging in Japanese encephalitis and their correlation with clinical profile in pediatric patients
}

\author{
Abrar S ${ }^{1}$, Sharma B ${ }^{2}$, Ansari M.J ${ }^{3}$, Bansal A ${ }^{4}$, Kushwaha K.P ${ }^{5}$, Rathi A.K ${ }^{6}$ \\ ${ }^{1}$ Dr Shahla Abrar, Senior Resident, ${ }^{2}$ Dr Bhoopendra Sharma, Associate Professor, ${ }^{3}$ Dr Mohammed Junaid Ansari, \\ Lecturer, ${ }^{4}$ Dr Abhishek Bansal, ${ }^{5}$ Dr K.P. Kushwaha, Profesor, ${ }^{6}$ Dr A.K. Rathi, Profesor, all authors are affiliated with \\ Department of Pediatrics, BRD Medical College Gorakhpur, UP, India.
}

Address for Correspondence: Dr. Shahla Abrar, MD Paediatrics, Senior Resident, Department of Paediatrics, BRD Medical College, Gorakhpur, India. Address- Type 3/19, BRD Medical College, Gorakhpur, India. Email id: shahlaabrar7@gmail.com

\begin{abstract}
Introduction: Japanese encephalitis is a major public health problem in Indian subcontinent. Regardless of all advances in prompt diagnosis of JE, it may be difficult to differentiate JE from other viral encephalitis. Aim: This study was done to know the topographic patterns of CT and MRI abnormalities in JE encephalitis. Methodology: This retrospective observational study was done in children 1-15 years of age who suffered from JE encephalitis diagnosed by MACELISA and in whom CT/MRI was done. Total 25 patients were enrolled. There CT/MRI findings were analysed and correlated with clinical features. Results: The finding were principally seen in thalamic (40\%; $\mathrm{n}=10)$ and basal ganglia $(24 \% ; n=6)$ in the form of hypo densities. Similar forms of lesions were also found in cortical region, Frontal=2 patients, parietal $=6$ patients, temporal $=7$ patients and occipital $=1$ patient. MRI was done in eleven patients. Our MRI findings were also in correlation with CT findings with most common being thalamic $(n=10)$ and basal ganglia $(n=5)$. Temporal $(n=4)$, parietal $(n=3)$ and occipital $(n=2)$ lobe changes. Conclusions: The imaging findings on CT and MR imaging evidence the pathologic changes. Majority of lesions on CT/MRI were in thalamus and basal ganglia, but in some cases cortical regions were also involved. Temporal involvement, which was previously a reflection of Herpes encephalitis on $\mathrm{CT} / \mathrm{MRI}$, can also be seen in JE encephalitis.
\end{abstract}

Keywords: Thalamus, Basal ganglia, Temporal, Dystonia.

\section{Introduction}

Japanese encephalitis virus (JEV) is a mosquito-borne flavivirus which causes significant epidemics of encephalitis throughout the world with 50,000 cases of encephalitis mostly affecting the children below 10 years of age causing 10,000 deaths annually [1, 2]. It has widespread distribution all over Asia and posing threat to many countries [3]. It is endemic in the Indian subcontinent, peculiarly in the north East Indian states of Assam, Eastern Uttar Pradesh and epidemics occur in summer rainy season, when there is ideal environment for mosquito to breed [4]. Case fatality ranges from $20 \%$ to $30 \%$ and up to $50 \%$ of those who recover are left with disabling

Manuscript received: $15^{\text {th }}$ July 2016

Reviewed: $27^{\text {th }}$ July 2016

Author Corrected; $10^{\text {th }}$ August 2016

Accepted for Publication: 22 ${ }^{\text {nd }}$ August 2016 neurologic deficit [5]. The diagnosis is commonly based on demonstrating a rising titre of antibodies against JE virus in acute and convalescent sera [6]. There are very few reports of CT and MRI changes in patients of Japanese encephalitis. In the face of all advances in quick diagnosis of JE, it may be difficult to differentiate JE from other viral encephalitis viz. herpes encephalitis. Furthermore, it is important to differentiate JE from other encephalitis for rational therapy in an endemic area like India.

This has an important bearing on future management of the patients. Although expensive, Neuroimaging is now readily available in most of the places and thus, its help can be sought in the diagnosis of encephalitis. This study was done to know the topographic patterns of CT 
and MRI abnormalities in JE encephalitis and to correlate the CT and MRI abnormalities with that of clinical features.

\section{Methodology}

This was a retrospective observational study done on children aged 1-15 years admitted in our institute in the months of June 2011 through August 2011 and were diagnosed as JE encephalitis. Thirty two patients were diagnosed as JE encephalitis during this period.

The diagnosis of JE was confirmed by positive immunoglobulin $\mathrm{M}$ (IgM) antibodies to JE virus (JEV) in the CSF by IgM capture enzyme-linked immunosorbant assay (MAC-ELISA) kit from NIV Pune. Twenty five patients with JE had undergone CT or MRI imaging or both and were enrolled for the study. MR imaging was done in 11 and CT in 25 . Rest all patients were excluded from the study.

Cranial computed tomography was carried out using a third generation scanner. Axial sections $(10 \mathrm{~mm})$ were obtained parallel to the orbitomeatal line. Cranial MRI was carried out on a $2 \mathrm{~T}$ scanner operating at $1.5 \mathrm{~T}$ (Magnetom, Siemens, Germany).

A detailed history and neurological examination was carried out in all the patients. The level of consciousness was assessed by the Glasgow coma scale. The extrapyramidal signs such as rigidity, dystonia, dyskinesia, and other abnormal movements were also noted on a precoded proforma. CT/MRI were analysed according to the site of lesion and findings were correlated with the clinical features.

\section{Results}

CT scan was carried out in four patients with acute illness and did not give any significant diagnostic finding helpful in the diagnosis and management of these patients.

However when CT is carried out after 10-15 days particularly in patients showing poor recovery and in those patients having extrapyramidal symptoms, CT scan abnormalities were seen in $80 \% \quad(n=20)$ cases.

The finding were principally seen in thalamic $(n=10)$ and basal ganglia $(n=6)$ in the form of hypo densities. Similar form of lesions was also found in cortical region. Frontal $=2$ patients, parietal $=6$ patients, temporal $=7$ patients and occipital $=$ 1 patient. It is striking to note that there was no CT scan changes found in mid-brain, Pons and cerebellum (Table: 1).

Table-1: CT findings in patients of JE encephalitis.

\begin{tabular}{|c|c|c|c|}
\hline CT Scan changes & Unilateral & bilateral & $\mathrm{n}(\%)$ \\
\hline & $\mathrm{n}(\%)$ & $\mathrm{n}(\%)$ & $05(20)$ \\
\hline NORMAL & - & - & $20(80)$ \\
\hline ABNORMAL & - & $7(28)$ & $6(24)$ \\
\hline Thalamus & $3(12)$ & $4(16)$ & - \\
\hline Basal ganglia & $2(8)$ & - & - \\
\hline Midbrain & - & - & - \\
\hline Pons & - & - & $2(8)$ \\
\hline Cerebellum & - & & $6(24)$ \\
\hline Cortical & $2(8)$ & - & $7(28)$ \\
\hline frontal & $6(24)$ & $1(4)$ & $1(4)$ \\
\hline parietal & $6(24)$ & - & $3(12)$ \\
\hline temporal & $1(4)$ & $3(12)$ & \\
\hline occipital & - & & \\
\hline Ventricular dilatation & & & \\
\hline
\end{tabular}


MRI was done in eleven patients. Our MRI findings were also in correlation with CT findings with most common being thalamic $(90 \%, \mathrm{n}=10)$ and basal ganglia $(45 \%, \mathrm{n}=5)$ (table 2$)$.

Temporal $(36 \%, n=4)$, parietal $(27 \%, n=3)$ and occipital $(18 \%, n=2)$ lobe changes were also noted in MRI of patients.

Table-2: MRI findings in patients of JE encephalitis.

\begin{tabular}{|c|c|c|c|}
\hline MRI changes & unilateral & bilateral & Total \\
\hline & $\mathrm{n}$ & $\mathrm{n}$ & $\mathrm{n}$ \\
\hline Normal & - & - & - \\
\hline Abnormal & - & - & 11 \\
\hline Thalamus & 2 & 8 & 10 \\
\hline Basal ganglia & 1 & 4 & 5 \\
\hline Midbrain & 1 & 1 & 2 \\
\hline Pons & - & 1 & 1 \\
\hline Cerebellum & - & 1 & 1 \\
\hline \multicolumn{4}{|l|}{ Cortical } \\
\hline Frontal & - & - & - \\
\hline Parietal & 3 & - & 3 \\
\hline Temporal & 3 & 1 & 4 \\
\hline Occipital & 2 & - & 2 \\
\hline Ventricular dilatation & - & 1 & 1 \\
\hline
\end{tabular}

In an attempt to correlate between presenting symptoms and lesion on CT scan we found that fever, convulsion and altered sensorium appears to be most consistent symptoms and present in $100 \%$ of patients irrespective of area of involvement on CT scan findings. Headache and vomiting were uniformly present in all patients with ventricular dilatation. (Figure 1)

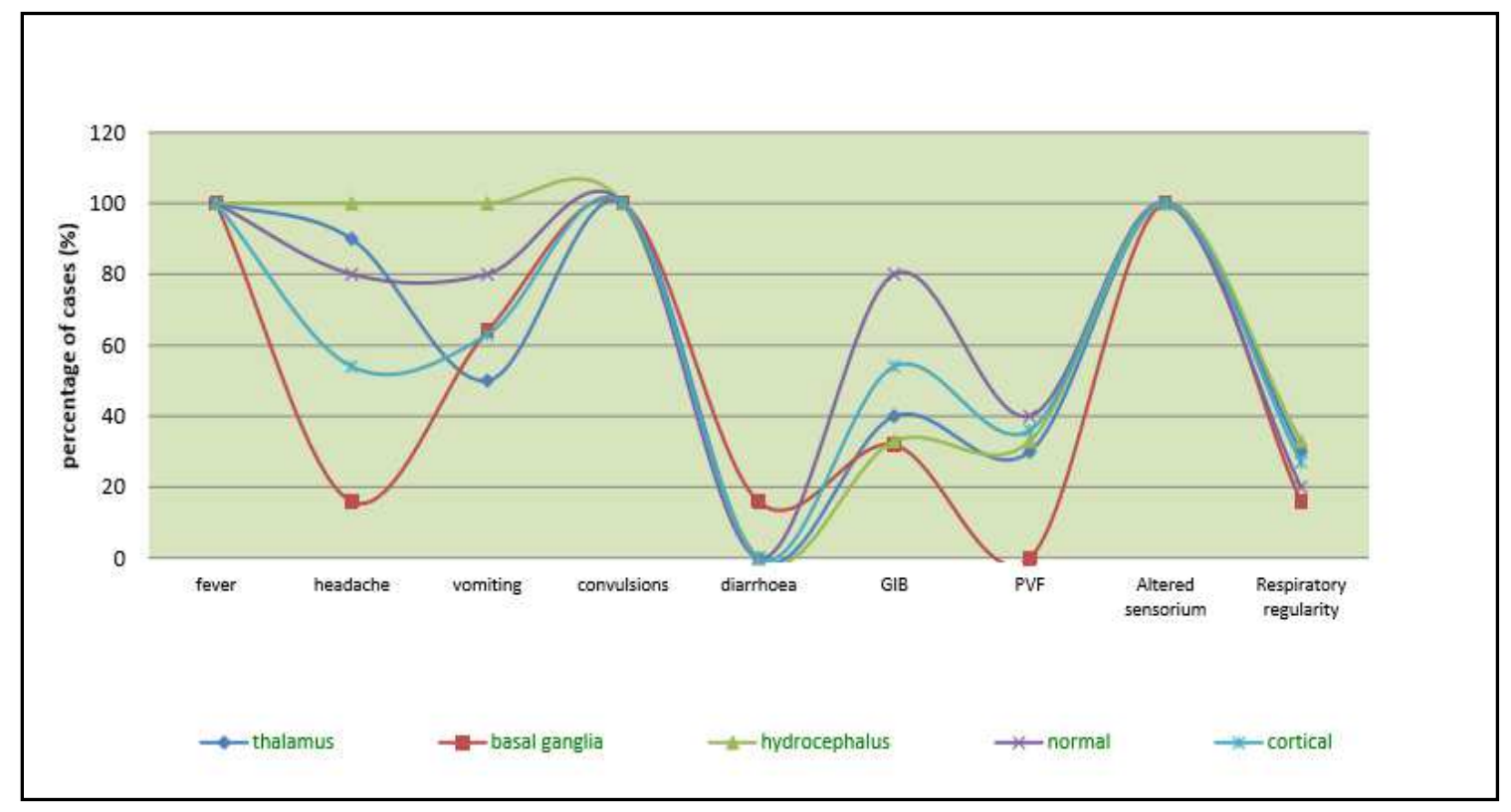

Figure-1: Correlation of CT findings with presenting symptoms in JE patients 
Hypertonia was present in $100 \%$ of patients with thalamic, basal ganglia lesions and patients with ventricular dilatation on CT scan. Cranial nerve palsy was most commonly found with thalamic lesion (30\%) and cortical lesion (28\%). Brisk deep tendon jerks were found in $100 \%$ patients in all except patients with cortical lesions in which they were found in $80 \%$ of cases. Power $<3 / 5$ in the form of hemiplegia or paraplegia was found in maximum $(66 \%)$ of patients with ventricular dilatation followed by patients with thalamic lesions (40\%). Power was apparently normal in all cases with normal CT scan. (Figure 2)

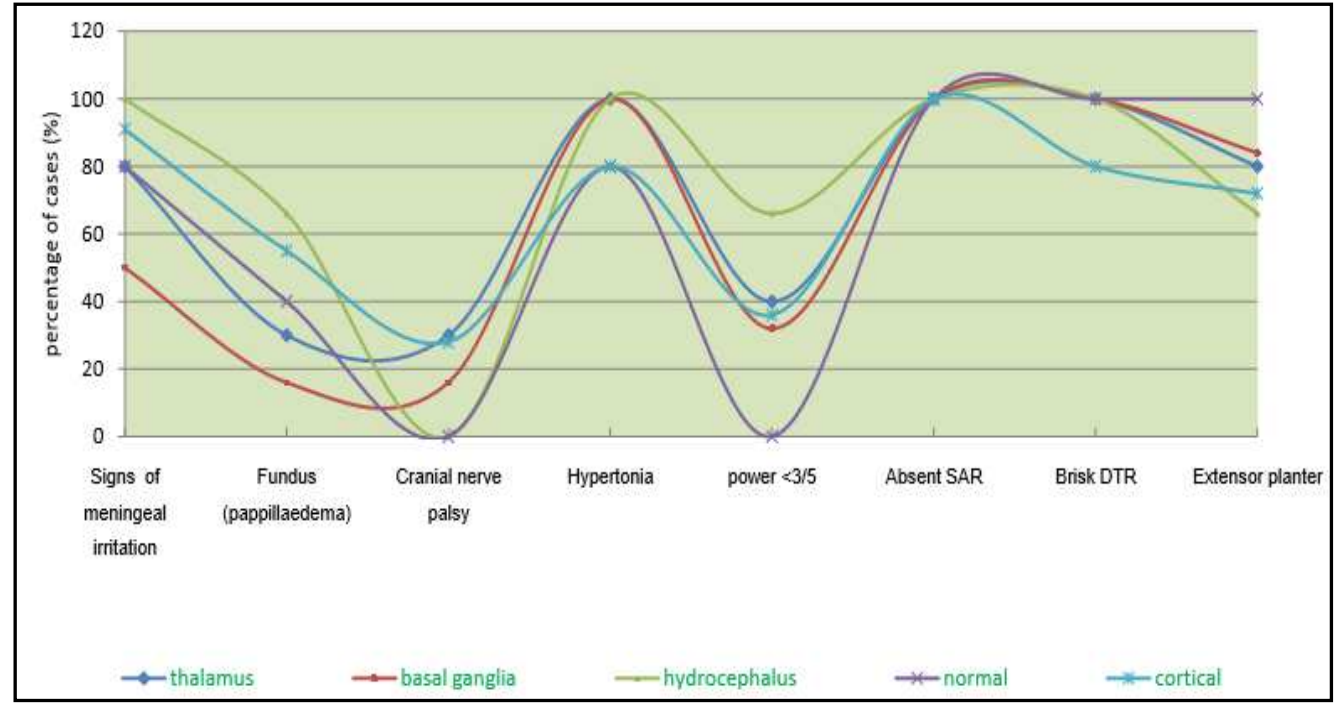

Figure-2: Correlation of CT findings with CNS examination in JE patients

Abnormal movements need special mention. In $76 \%$ of patients, where CT scan was carried out had abnormal movements in the form of dystonia, dyskinesia, chorea, tremor, athetosis, Parkinsonism, mixed and ill-defined. Dystonia was universally present in patients with thalamic lesions on CT scan, whereas $84 \%$ of patients with basal ganglia lesions had dystonia. Though dystonia was seen in all patients who had ventricular dilatation, it was important to note that all these patients also had accompanying thalamic lesions along with ventricular dilatation. Tremor was found in patients with thalamic involvement. However, it was also seen in patients with ventricular dilatation and cortical lesions. It might be due to the fact that in all such patients thalamic lesions were also associated. Chorea was most commonly present with thalamic lesion (30\%) followed by solitary cortical lesion (16\%). (Figure 3)

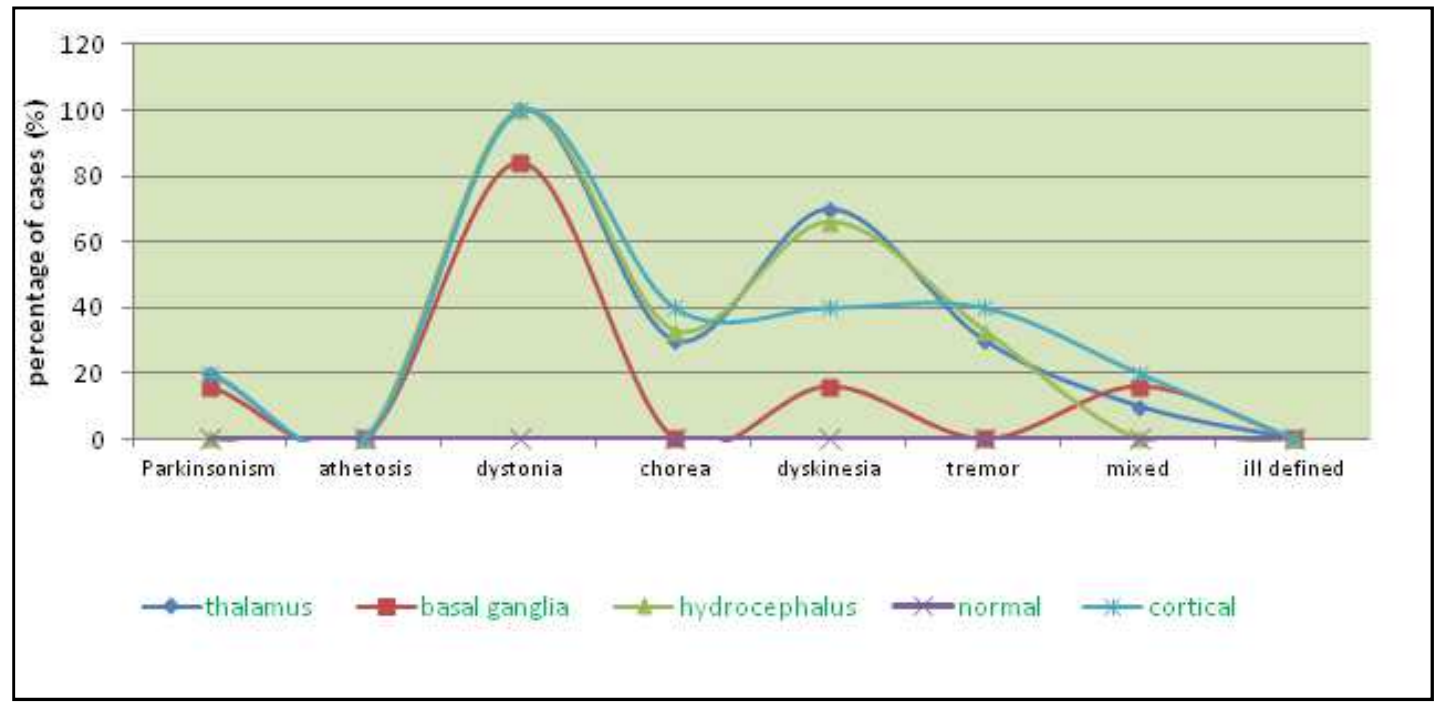

Figure-3: Correlation of CT findings with abnormal movements in JE encephalitis patients 


\section{Discussion}

Uttar Pradesh in India is an economically poor state and is an epicentre of JE [7]. Though, we need CT and MRI findings to evaluate these patients for their better management, it has been difficult task so far because of economic constrains.

In the present study, we have mobilized our own resources in addition to those who could afford to have CT and MRI of these patients.

The brunt of abnormalities in this retrospective observational study was noted in the form of thalamic and basal ganglia hypo densities. Our findings were in line with Mishra et al and Kalita et al, though the incidence of the abnormalities in their patients was much lower $[8,9]$.

We did not found any abnormalities in brain stem and cerebellum, this was in contrast to the findings of Mishra et al and Kalita et al. Cortical changes in the form of hypo densities in various area were observed in 11 patients. Kalita et al reported cortical involvement in $18 \%$ and Mishra et al reported in $7 \%$ of their patients $[8,9]$.

Pathologic transformation in the brains of acute JE patients are characterized by glial nodules and circumscribed necrolytic foci and involve the thalami, substantia nigra $(\mathrm{SN})$, corpus striatum, cerebral cortex, brain stem, and cerebellum. White matter areas may occasionally be involved [7].

The imaging findings on CT and MRI reflect the pathologic changes. The most consistent finding previously reported in JE encephalitis is bilateral thalamic lesions with or without hemorrhagic changes on MR imaging. Lesions are also noted in the substantia nigra, brain stem, cerebellum, cerebral cortex, and white matte $[10,11]$.

Nearly one third of JE patients in our study showed temporal lobe involvement in CT or MRI, a finding more characteristic of Herpes simplex encephalitis. Some imaging studies have found temporal lobe involvement in patients. Seventeen percent of patients had temporal lobe involvement in one study [12].
In another, only one patient of 43 showed temporal lobe involvement [11]. The JE virus invades the nervous system through the hematogenous route, though subsequent spread of the virus seems to occur along the dendritic axonal processes [13].

There is often sharing of blood supply by parts of the thalamus, cerebral peduncles, hippocampus, and uncus, mainly through the anterior choroidal, lateral posterior, and medial posterior choroidal arteries [14, 15].

These arteries have reciprocal vascular supply. This may explain concurrent involvement of the temporal lobe along with the thalami in our patients.

It is interesting to note that although all 11 patients in our study showed lesions on MR imaging scans, 20 of 25 showed the lesions on CT. More lesions were seen on MR imaging than on CT.

This highlights the usefulness of MR imaging over CT in the evaluation of JE. Other studies have also highlighted usefulness of MR imaging over CT in detecting JE lesions [11].

In the present study, $76 \%$ of patients with JE, where CT/MRI was carried out, developed movement disorders in the acute or subacute stage of the illness.

Most common movement disorder observed was dystonia. Focal central nervous system lesions affecting lentiform or caudate nuclei, particularly those disrupting the striatopallidothalamocortical pathway may cause dystonia [16].

A study done using positron emission tomography in hemidystonic patients with basal ganglia or thalamic lesions demonstrated metabolic overactivity in frontal association areas when moving the affected limb [17]. Dystonia after thalamic stroke has been reported after 1-9 months of seizure [18].

In our study, dystonia was universally present in patients with thalamic or basal ganglia lesions on CT scan. However, some of the JE patients with dystonia had cortical involvement in addition to thalamic lesions. 


\section{Conclusion}

Approximately one third of the JE encephalitis patients die and half of the survivors suffer severe neuropsychiatric sequelae from the disease [5]. Although a histopathological examination is a helpful tool for the diagnosis of JEV, it is not clinically applicable.

The current diagnostic methods for JEV rely on JEV IgM detection by ELISA and JEV RNA sequence detection by PCR.

However, these methods are time-consuming and offer no timely guidance for clinical decisions. Characteristic Neuroimaging findings could be key for early differentiation

Acknowledgements- The author acknowledge the favourable input of Prof. K.P. Kushwaha and Prof A.K. Rathi, Department of paediatrics, BRD medical college in guiding during different phases of study.

\section{Funding: Nil, Conflict of interest: Nil \\ Permission from IRB: Yes}

\section{References}

1. Saxena S.K. Japanese encephalitis: perspectives and new developments. Future Neurol. 2008;3(5): 515-521.

2. Diagana M, Preux PM, Dumas M. Japanese encephalitis revisited. J Neurol Sci. 2007 Nov 15;262 (1-2):165-70. Epub 2007 Jul 23.

3. Yamanaka A, Mulyatno KC, Susilowati H, Hendrianto E, Utsumi T, Amin M, Lusida MI, Soegijanto S, Konishi E. Prevalence of antibodies to Japanese encephalitis virus among pigs in Bali and East Java, Indonesia, 2008. Jpn J Infect Dis. 2010 Jan;63 (1):58-60.

4. van den Hurk AF, Ritchie SA, Mackenzie JS. Ecology and geographical expansion of Japanese encephalitis virus. Annu Rev Entomol. 2009;54:17-35. doi: 10.1146/annurev.ento.54.110807.090510.

5. Campbell GL, Hills SL, Fischer M, Jacobson JA, Hoke CH, Hombach JM, Marfin AA, Solomon T, Tsai TF, Tsu VD, Ginsburg AS. Estimated global incidence of Japanese encephalitis: a systematic review. Bull World Health Organ. 2011 Oct 1;89(10):766-74, 774A774E. doi: 10.2471/BLT.10.085233. Epub 2011 Aug 3.

6. A Mathur, K L Arora, S Rawat, and U C Chaturvedi. "Persistence, latency and reactivation of Japanese encephalitis virus infection in mice," Journal of General Virology. 1986;67:381-385.

7. Saxena SK, Singh M, Pathak AK, Mathur A. Reply to 'Encephalitis outbreak finds Indian officials unprepared'. Nat Med. 2006 Mar;12(3):269-70.

8. Kalita J, Misra UK. Comparison of CT scan and MRI findings in the diagnosis of Japanese encephalitis. J Neurol Sci. 2000 Mar 1;174(1):3-8.

9. Kalita J, Misra UK, Pandey S, Dhole TN. A comparison of clinical and radiological findings in adults and children with Japanese encephalitis. Arch Neurol. 2003 Dec;60(12):1760-4.

10. Shoji H, Kida H, Hino H, Matsuura S, Kojima K, Abe T, Utsunomiya H, Okada Y, Nakamura Y, Okudera T. Magnetic resonance imaging findings in Japanese encephalitis. White matter lesions. J Neuroimaging. 1994 Oct;4(4):206-11.

11. Kalita J, Misra UK. Comparison of CT scan and MRI findings in the diagnosis of Japanese encephalitis. J Neurol Sci. 2000 Mar 1;174(1):3-8.

12. Handique SK, Das RR, Barman K, Medhi N, Saharia B, Saikia P, Ahmed SA. Temporal lobe involvement in Japanese encephalitis: problems in differential diagnosis. AJNR Am J Neuroradiol. 2006 May;27(5):1027-31.

13. Gourie-Devi M, Ravi V, Shankar SK. Japanese encephalitis. An overview.In: Clifford Rose, ed. Recent advances in tropical neurology. Amsterdam: Elsevier Science B.V;1995:217-235.

14. Mohr JP, Steinke W, Timsit SG, Sacco RL, Tatemichi TK. The anterior choroidal artery does not supply the corona radiata and lateral ventricular wall. Stroke. 1991 Dec;22(12):1502-7. 
15. Osborne AG, Davis WL, Jacobs J. Normal vascular anatomy. In: Osborne $\mathrm{AG}$, ed. Diagnostic neuroradiology. St. Louis, Mo: Mosby-Year Book, Inc.; 1994:117-53.

16. Bressman SB, de Leon D, Raymond D, Greene PE, Brin MF, Fahn S, Ozelius LJ, Breakefield XO, Kramer PL, Risch NJ. Secondary dystonia and the DYTI gene. Neurology. 1997 Jun;48(6):1571-7.
17. Ceballos-Baumann AO, Passingham RE, Marsden CD, Brooks DJ. Motor reorganization in acquired hemidystonia. Ann Neurol. 1995 Jun;37(6):746-57.

18. Wooten GF, Lopes MB, Harris WO, Reagan TJ, Vandenberg SR. Pallidoluysian atrophy: dystonia and basal ganglia functional anatomy. Neurology. 1993 Sep;43(9):1764-8.

\section{How to cite this article?}

Abrar S, Sharma B, Ansari M.J, Bansal A, Kushwaha K.P, Rathi A.K. Neuroimaging in Japanese encephalitis and their correlation with clinical profile in pediatric patients. Int J Pediatr Res.2016;3(8):617-623.doi:10.17511/ijpr.2016.i08.11 